\title{
BMI open Protocol for an experimental study design to evaluate computer-enabled intervention to prevent and manage metabolic syndrome
}

\author{
Ashish Joshi, ${ }^{1,2}$ Shruti Mehta, ${ }^{2,3}$ Kandarp Talati, $^{2}$ Ashoo Grover ${ }^{4}$
}

To cite: Joshi A, Mehta S, Talati K, et al. Protocol for an experimental study design to evaluate computer-enabled intervention to prevent and manage metabolic syndrome. BMJ Open 2013;3:e002163. doi:10.1136/bmjopen-2012002163

- Prepublication history for this paper are available online. To view these files please visit the journal online (http://dx.doi.org/10.1136/ bmjopen-2012-002163).

Received 27 September 2012 Revised 30 October 2012 Accepted 1 November 2012

This final article is available for use under the terms of the Creative Commons Attribution Non-Commercial 2.0 Licence; see http://bmjopen.bmj.com

\section{${ }^{1}$ Department of Health} Services Research Administration, Center for Global Health and

Development, College of Public Health, University of Nebraska Medical Center, Omaha, Nebraska, USA ${ }^{2}$ Center for Public Health Informatics, AIPH, Bhubaneswar, Orissa, India ${ }^{3}$ Foundation of Healthcare Technologies Society, New Delhi, India

${ }^{4}$ Department of Health Research, Indian Council of Medical Research,

Government of India, New Delhi, India

\section{Correspondence to} Dr Ashish Joshi; ashish.joshi@unmc.edu

\section{ABSTRACT}

Introduction: The rising prevalence of overweight and obesity has a direct correlation with increasing prevalence of hypertension, dyslipidaemia, type 2 diabetes, metabolic syndrome (MetS) and cardiovascular diseases. Most of the previous studies have been cross-sectional in nature and have looked at the prevalence of metabolic syndrome. Despite the clinical and public health importance of this phenomenon, not enough work has been carried out so far to study and remedy this situation. The objectives of the proposed study is to develop an innovative usercentred informatics platform that will facilitate delivery of a multifactorial intervention after taking into account user sociodemographics, health behaviour, prior disease state and knowledge attitudes and practices.

Objective: The objective of the proposed study is to develop an innovative user-centred informatics platform that will facilitate delivery of a multifactorial intervention after taking into account users' sociodemographics, health behaviour, prior disease state and knowledge, attitudes and behaviour.

Methods and analysis: A randomised two-group repeated-measures clinical trial design will be used, on 750 subjects from urban, rural and slum areas, in an Indian setting. The study participants will be randomly assigned to either the intervention (computer-based MetS Program, CBMP) or control (printed educational material, PEM) group. Both the groups will undergo screening, learning and evaluation assessments at the time of the visit and at follow-up visits 30,60 and 90 days after the first visit.

Outcomes: The outcomes expected in the intervention group include improvement in Mets-related knowledge, adherence to self-care practices, better quality of life and increased satisfaction with medical care.

Ethics and dissemination: The study has been approved by the Institutional Review Board of Asian Institute of Public Health (IRB\#621). The proposed study will also help us assess the usefulness and challenges of technology to disseminate health education among diverse users. Findings will be disseminated through peerreviewed publications and national and international conference presentations to various stakeholders and local community health leaders. The ClinicalTrials.gov Identifier is NCT01713465.

\section{ARTICLE SUMMARY}

\section{Article focus}

- The study aims to describe the role of information technology to improve self management of metabolic syndrome among individuals living in urban, rural and slum settings.

Key message

- Computers can be a useful medium to increase awareness about metabolic syndrome.

Strengths and limitations of this study

- First study to explore the impact of informatics interventions.

- Prospective experimental study design.

- Develop in-depth understanding about the various risk factors that impact poor outcomes of metabolic syndrome in individuals living in diverse Indian settings.

- Further study facilitates an interactive bilingual education program.

- More research is needed to explore the impact of informatics interventions on long term clinical outcomes.

- A study with a long follow up is needed. The existing study does not compare the impact of the interventions in reducing the costs of the healthcare.

\section{INTRODUCTION}

Non-communicable diseases is a barrier to developmental goals that include poverty reduction, health equity, economic stability and human security. ${ }^{1}$ The rising prevalence of overweight and obesity has a direct correlation with the increasing prevalence of obesity-related comorbidities: hypertension, dyslipidaemia, type 2 diabetes mellitus, metabolic syndrome (MetS) and cardiovascular disease (CVD). ${ }^{2}$ CVDs are the causes of death rates in $40 \%$ of urban Indians and $30 \%$ of rural Indians and this trend has shown an exponential increase over time. ${ }^{3}$ MetS is a widely prevalent and multifactorial disorder characterised by diabetes or 
high fasting glucose, central obesity, abnormal cholesterol and triglyceride levels and hypertension. ${ }^{4}$ Over the years several classifications for the MetS have been proposed; the major definitions used are the WHO,${ }^{5}$ European Group for the Study of Insulin Resistance (EGIR), ${ }^{6}$ National Cholesterol Education Program Adult Treatment Panel (NCEP ATP III), ${ }^{7}$ American Association of College of Epidemiology (AACE), ${ }^{8}$ National Heart, Lung and Blood Institute/American Heart Association (NHLBI/AHA) ${ }^{9}$ and International Diabetes Federation (IDF). ${ }^{10}$

India is a major contributor to the global increase in cardiovascular disease through the increased mortality and prevalence of MetS. The incidence of this syndrome has been estimated to increase with age for individuals over 50 years of age. ${ }^{11}$ Various studies from around the world, including those of the general population, indicate that individuals aged 20-25 and above have a prevalence of $24 \%$ (India), 28\% (USA), 30.1\% (Tehran), $33.4 \%$ (Turkey) and 39.3\% (Saudi Arabia). Studies have shown that one-fourth to one-third of the urban population of India has MetS. ${ }^{12}$ MetS is highly prevalent among urban populations of Indians (35.2\% vs 20.6\%) compared to rural populations. Its prevalence increases with age and is 1.5-2 times higher in women than in men. ${ }^{12}{ }^{13}$ Most of the previous studies have been crosssectional in nature and have looked at prevalence rather than come up with an intervention model to prevent, monitor and manage MetS. ${ }^{11-13}$ Despite the clinical and public health importance of this phenomenon, not enough work has been carried out so far to study and remedy this situation.

The objective of the proposed study is to develop an innovative user-centred informatics platform that will facilitate delivery of a multifactorial intervention after taking into account users' sociodemographics, health behaviour, prior disease state and knowledge, attitudes and behaviour (KAB). To our knowledge, this is the first study to design and develop an intervention model after assessing the needs of users from diverse settings including urban, rural and slum areas in an Indian context.

The proposed study has three aims:

- To explore the multifactorial needs of a diverse group of individuals at risk of MetS in an Indian setting.

- To design and develop an interactive, tailored, usercentred and multifactorial, technology-mediated, evidence-based intervention to prevent and manage MetS in an Indian setting.

- To evaluate a prototype aimed at facilitating MetS intervention among the diverse group of users including urban, rural and slum individuals in an Indian setting.

\section{METHODS AND ANALYSIS}

Study design

- A randomised two-group repeated measure clinical trial design will be used.
Study settings

We will recruit subjects from the following sites:

- SCB Medical College Cuttack: urban population.

- Other affiliate hospitals in Bhubaneswar: mixed population tertiary care patients (urban as well as referred cases from rural areas).

- Urban slums, Bhubaneswar, India.

We have collaborated with the local community health workers and healthcare practitioners at different urban, rural and slum sites to ensure that we have adequate enrolment as proposed.

\section{Subject eligibility}

The inclusion criteria include: (1) adults aged 30 years and above, (2) agreeing to participate in the study and (3) being available for follow-up interview. Subjects will be excluded if any of the aforementioned criteria are not met including the following: (1) mental and physical challenges that make it difficult to use the touch-based computer program, (2) unavailability for telephone follow-up and (3) involvement in other clinical trials or protocols related to MetS.

\section{Study groups}

The study participants will be randomly assigned to either the intervention (computer-based MetS Program CBMP) or control (Printed Educational Material, PEM) groups. The randomisation will be done at the time the participant comes to the outpatient clinics in these diverse settings. A computer-generated system that will follow 1:1 allocation at each site including urban, rural and slum areas for allocating the individuals either in the intervention or the control group will be utilised. Both the groups will undergo screening, learning and evaluation assessments at the time of the visit. Apart from that, study participants in both the groups need to come for follow-up visits 30,60 and 90 days from the first visit for ongoing education. The outcome assessors and data analysts will be blinded after assignment to interventions as the data analysis sheet will not have the identifier that will indicate which individual was in the intervention or the control group.

A detailed outline of the intervention is described below.

\section{Patient education and motivation tool}

We will modify and adapt the existing Patient Education and Motivation Tool (PEMT), a touch screen, computerbased interactive health education program. PEMT is designed based on a variety of cognitive-behavioural theories ${ }^{14}$ and aimed at delivering health education to prevent and manage MetS among diverse users. PEMT facilitates health information and messages to be adapted depending on the psychosocial elements including attitude, self-efficacy, expectations, personal norms and social influence. ${ }^{15-17}$ The multimedia information presented will be in multilingual format (English 
AND Oriya, an Indian language). PEMT has three key components including:

\section{Screening component}

This will include age (years), gender, years of education, income, employment and insurance status. Information on MetS knowledge and attitudes and practice, quality of life and satisfaction to medical care will also be assessed.

\section{Learning component}

Using self-report responses of the individuals in the intervention group (CBMP), the decision logic and algorithm will process the information and deliver tailored MetS educational messages. For example, if an individual has a family history of diabetes and no other risk factors, the individual will be provided feedback on the preventive measures that he/she needs to have to maintain himself in a healthy condition. If a study participant indicates that he/she does not perform any physical activity, then the system would recommend to them the exercise suggestions and provide tailored health information about the short-term and long-term benefits of physical activity.

\section{Evaluation component}

Change in MetS knowledge, attitudes and practice (KAP), quality of life, adherence to treatment and satisfaction to medical care will be assessed in study participants in both the groups. Appropriate feedback will be given in the form of reinforced educational messages and encouragement and motivational prompts for those using the touch screen computer-based program.

\section{PEMT interaction metrics}

Another important aspect of PEMT is that it captures an individual's interactions with this interactive educational support program and generates usage metrics. ${ }^{16}$ Usage is clearly an important characteristic in assessing the popularity of a computer-mediated health education programme. The logs provide data on what people have done, which gives logs their strength and differentiates them from other data capture methods like interviews and questionnaires. Earlier studies have shown that PEMT has improved asthma, influenza and breastfeeding KAP among different users in diverse settings.

\section{Procedures for implementing intervention (CBMP)}

The touch screen computer-based MetS educational support program will be developed by modifying existing PEMT as described earlier. This modification will include several stages.

- MetS content finalisation, decision rules and PEMT modification design: We will have in-depth interviews to finalise the content, logic and functionality of the computerbased program. Forty individuals from urban, rural and slum settings will be enrolled, and the feedback acquired from them will help us to identify the topics that the study participants will perceive as useful to manage MetS. Both qualitative and quantitative data will be gathered using a series of open-ended and close-ended questions to identify the educational modules relevant to the user. The educational content developed will then be finalised in two languages including English and Oriya (a local Indian dialect). The educational material will have related audio and images to account for the health literacy levels of the individuals.

- BMP pretesting using heuristic evaluation: The prototype designed will undergo a series of usability assessments throughout the development process. Heuristic evaluations, which are often referred to as 'expert reviews' or 'usability audits', are an efficient way of assessing the usability of information technologies and are particularly useful early in the development process when it is least expensive to make changes. Nielsen's heuristics are probably the most used usability heuristics for user interface design. The 10-item assessment is given a rating on a scale of $0-4$, where 0 means no usability problem at all, 1 means a cosmetic problem, 2 means a minor problem, 3 means a major problem and 4 means a catastrophic problem.

- Usability assessments using user testing: We will identify representative tasks including both critical and highfrequency activities. We will recruit 10 study participants each at the different study sites to interact with the system. The results of the user testing will help incorporate the recommended changes in the design of CBMP. A system usability scale is a 10-item questionnaire with five response options ranging from strongly disagree to strongly agree.

- Study enrolment and data collection procedures: Baseline and follow-up data will be collected at months 1, 2, 6, 9 and 12 resulting in five data collection time periods overall. Baseline evaluation will be performed during the first outpatient visit at the hospital sites in urban, rural and slum settings (table 1 ). Periodic reminders will be made to the study participants by fliers and through community health workers and healthcare practitioners.

\section{Informed consent}

The field staff will administer informed consent after the eligible subjects have agreed to participate in the study. The study has been approved by the Institutional Review Board IRB NO-621/AIPH/BBSR.

\section{Data entry and quality assurance}

Data will be entered into a relational database structure using Microsoft Access. To ensure efficiency and highquality data collection and processing, we will have (1) a well-trained team of field workers, (2) a clearly defined study manual, (3) weekly meetings with the research staff, (4) logs of all study participant contacts, (5) logs of all the data instruments filled during each 


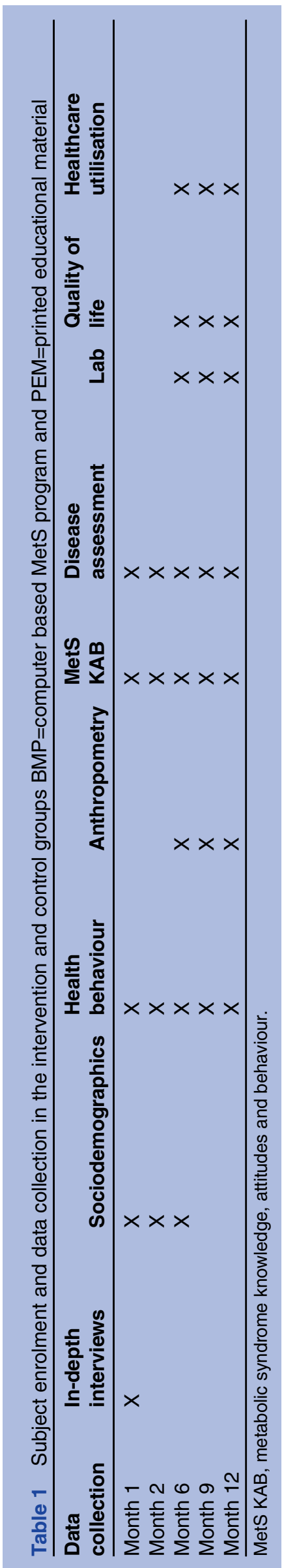

visit for every patient, (6) central data processing and (7) weekly data checks. Security of data will be maintained through regular backups and all computers and specific data files will be password protected and kept in a locked file cabinet. The information will be accessed only by those personnel who will be part of the research team. All data will be stored in a passwordprotected computer in a locked office of the principal investigator for 3 years from the point of study completion at which time they will be destroyed. Security of the data will be maintained through regular backups, and all computers and specific data files will be password protected and kept in a locked file cabinet.

\section{Data collection instruments} In-depth interviews

We will conduct in-depth interviews with 40 people each from urban, rural and slum areas. The optimum number of participants for an in-depth interview is typically $6-10$ people with similar backgrounds. ${ }^{18}$ These interviews will help us examine factors that play a pivotal role in the prevention and management of MetS.

\section{Potential confounders}

- Sociodemographics: They include age, income levels, employment status, education level and smoking and alcohol status. Information is also gathered about prior use of computers, its frequency of usage, prior use of internet and sources of health information. The three health literacy screening questions, which were performed optimally in a previous study, will be used. $^{19}$

- Anthropometry: Height and weight will be collected using a standard technique ${ }^{20}$ and body mass index will be computed from these measurements.

- MetS KAB: We will utilise a previously studied KAB questionnaire. $^{21}$ The $\mathrm{KAB}$ has been proposed as a model to explain how the accumulation of knowledge leads to a change in attitude and ultimately to a change in behaviour. ${ }^{22}$

- Self-report disease assessment: Self-report disease assessment will be performed to assess the presence of diabetes, hypertension or any other cardiovascular disease.

- Laboratory assessment: These include fasting blood glucose levels and lipid profile.

\section{Outcomes}

- Adherence to recommendations: Comparisons will be made between the intervention and the control groups to determine the number of study participants that sought reassessment of their risk factors (such as blood pressure, blood sugar and hypercholesterolaemia monitoring), diet and physical activity recommendations.

- Quality of life: We will use the WHO brief version of quality of life questionnaire (WHOQoL-BREF) that contains a total of 26 questions, based on four 
Table 2 Analysis plan

\begin{tabular}{lll}
\hline Variables assessment & Variable types & Data analysis \\
\hline In-depth interviews & Qualitative & Content analysis using NVivo software \\
Sociodemographics & Continuous and categorical & T and $\chi^{2}$ tests \\
Health behaviour & Continuous and categorical & T and $\chi^{2}$ tests \\
Anthropometry & Continuous & T test \\
MetS KAB & Continuous & General linear mixed model \\
Disease assessment & Continuous & General linear mixed model \\
Laboratory assessment & Categorical & $\chi^{2}$ test \\
Outcomes & Continuous and categorical & T $\chi^{2}$ tests \\
Quality of life & Categorical & $\chi^{2}$ test \\
Satisfaction to medical care & Continuous & General linear mixed model \\
Healthcare utilisation &
\end{tabular}

domains of physical health, psychological health, social relationship and environmental issues. ${ }^{23}$

- Satisfaction to medical care: We will use client satisfaction questionnaire (CSQ-8), ${ }^{24}$ an eight-item questionnaire to assess the level of subject satisfaction with medical care.

- Healthcare utilisation: This will include self-report information about the number of emergency room visits, number of doctor visits and number of hospital admissions over a 6 -month period.

\section{Analysis plan}

\section{Sample size justification}

We plan to enrol 750 patients (250 each from the urban, rural and slum sites) and equally randomised to control and intervention groups. For a log-rank test with a twosided significance level of 0.05 , assuming uniform accrual with an accrual time of 24 weeks and a follow-up time of 12 weeks, a sample size of 125 per group is required to obtain a power of at least 0.8 . The sample size will be increased to 862 study participants to account for anticipated attrition of $15 \%$.

\section{Statistical analysis}

We will present a table to summarise the overall characteristics of all variables. The distributions of continuous outcomes will be explored for normality and transformations will be used if warranted. Continuous variables will be summarised using means and SD while frequencies will be reported for categorical variables. We will crosstabulate the intervention group assignments against outcome variables to assess the direction and degree of association in this initial stage. A multivariate regression analysis will be performed to examine the variables that impact improvement in MetS-related KAP.

All primary analyses will be evaluated based on data from this population on an intention-to-treat basis according to the intervention group they were assigned to at randomisation. Baseline characteristics will be summarised by the intervention group. A generalised linear mixed model using a binomial distribution and logit link function will be used to examine binary outcomes, with the intervention group as a fixed effect and the study participant as a random effect using a Variance Components covariance structure. Covariates will be added to the model to adjust for confounders that differ significantly between the two groups at the 0.20 level of significance (table 2).

\section{Project timelines and milestones}

The research plan entails the performance of the tasks in table 3 .

\section{ETHICS AND DISSEMINATION}

To our knowledge, the study is the first of its kind that aims to develop a technology-mediated multifactorial intervention to prevent and manage MetS in an Indian setting. The proposed research study will help us examine variables that influence the prevalence of MetS among individuals living in urban, rural and slum settings. Further, the feedback received through the in-depth interviews will help to design and develop a user-centred informatics platform that can deliver

\section{Table 3 Project timeline}

\begin{tabular}{|c|c|}
\hline Tasks involved & Timeline \\
\hline Research team meet & Month 1 \\
\hline In-depth interviews & Months 1 and 2 \\
\hline MetS educational content developed & Month 2 \\
\hline $\begin{array}{l}\text { Modify educational content and } \\
\text { existing PEMT }\end{array}$ & Months 3 and 4 \\
\hline $\begin{array}{l}\text { Computer program redesign using } \\
\text { heuristic principles }\end{array}$ & Months 3 and 4 \\
\hline User testing & Month 5 \\
\hline System deployment & Month 6 \\
\hline Subject recruitment & Months 6-9 \\
\hline Data collection & Months 6-12 \\
\hline Analysis and results & Month $6,9,12$ \\
\hline Dissemination & Month $6,9,12$ \\
\hline
\end{tabular}


multimedia-driven health educational modules tailored to meet the needs of the diverse users in varied settings. The proposed study will also help us assess the usefulness of technology to disseminate health education among diverse users. The study will also help us identify challenges in the implementation of technology-mediated health interventions in different Indian settings.

Findings will be disseminated through peer-reviewed publications and national and international conference presentations. The study findings associated with every specific aim will be disseminated on time to various stakeholders involving the funding agency, Indian Council of Medical Research, Government of India, Divisions of Non-communicable Diseases and Rural Health, Ministry of Health and Family Welfare, Government of India. Findings of the studies will also be disseminated to the local community health leaders.

Contributors AJconceived the idea of the study and was responsible for the design of the study. AJ and SM are responsible for defining the elements of the intervention, and SM and KT were responsible for identifying the gaps in the existing literature review on metabolic syndrome. The initial draft of this manuscript was prepared by AJ and SM and was then circulated repeatedly among all authors for critical revision. AG provided her feedback on the methodology write-up section of this manuscript. AJ was the PI and lead writer. SM was the research coordinator of the study, KT was the analyst of the data and $A G$ reviewed the findings of the study. AJ, SM and KT helped plan the study, evolve the analysis plans and interpret the data; AG, along with the others, helped revise successive drafts of the manuscript.

Funding Funded by the Department of Health Research, Indian Council of Medical Research, New Delhi, India.

Competing interests None.

Ethics approval Asian Institute of Public Health, Bhubaneswar.

Provenance and peer review Not commissioned; internally peer reviewed.

Data sharing statement Data about the sociodemographics and knowledge attitudes and practices, adherence and quality of life can be made available by contacting Dr AJ (PI of the grant) through email at ashish1875@gmail.com.

\section{REFERENCES}

1. Grundy SM. Metabolic syndrome pandemic. Arterioscler Thromb Vasc Biol 2008;28:629-36.

2. Poirier P, Giles TD, Bray GA, et al. Obesity and cardiovascular disease: pathophysiology, evaluation, and effect of weight loss. Circulation 2006;113:898-918.

3. Enas EA, Senthilkumar A. Coronary artery disease in Asian Indians: an update and review. Coronary artery disease: risk promoters, pathophysiology, and prevention. Vol 1. New Delhi: Jaypee, 2005:21-57.

4. Cameron AJ, Shaw JE, Zimmet PZ. The metabolic syndrome: prevalence in worldwide populations. Endocrinol Metab Clin North Am 2004;33:351.
5. Duvnjak L, Duvnjak M. The metabolic syndrome-an ongoing story. J Physiol Pharmacol 2009;60(Suppl 7):19-24.

6. Balkau B, Charles M. Comment on the provisional report from the WHO consultation. European Group for the Study of Insulin Resistance (EGIR). Diabet Med 1999;16:442.

7. Alberti K, Zimmet $P$, Shaw J. Metabolic syndrome-a new world-wide definition. A consensus statement from the international diabetes federation. Diabet Med 2006;23:469-80.

8. Einhorn D, Reaven GM, Cobin RH, et al. American College of Endocrinology position statement on the insulin resistance syndrome. Endocr Pract 2003;9:237-52.

9. Grundy SM, Brewer HB Jr, Cleeman JI, et al. Definition of metabolic syndrome report of the National Heart, Lung, and Blood Institute/ American Heart Association Conference on scientific issues related to definition. Circulation 2004;109:433-8.

10. Expert Panel on Detection, Evaluation, and Treatment of High Blood Cholesterol in Adults. Executive summary of the third report of the National Cholesterol Education Program (NCEP) expert panel on detection, evaluation, and treatment of high blood cholesterol in adults (Adult Treatment Panel III). JAMA 2001;285:2486-97.

11. Gupta A, Gupta V. Metabolic syndrome: what are the risks for humans. Biosci Trends 2010;4:204-12.

12. Misra A, Khurana L. The metabolic syndrome in South Asians: epidemiology, determinants, and prevention. Metab Syndr Relat Disord 2009;7:497-514.

13. Reddy KS, Prabhakaran D, Chaturvedi V, et al. Methods for establishing a surveillance system for cardiovascular diseases in Indian industrial populations. Bull World Health Organ 2006:84:461-9.

14. Joshi A, Arora M, Dai L, et al. Usability of a patient education and motivation tool using heuristic evaluation. J Med Internet Res 2009;11:e47.

15. Joshi A, Lichenstein R, King J, et al. Evaluation of a computer-based patient education and motivation tool on knowledge, attitudes, and practice towards influenza vaccination. IEJHE 2009;12:1-15.

16. Joshi A, Lichenstein R, Rafei K, et al. A pilot study to evaluate self initiated computer patient education in children with acute asthma in pediatric emergency department. Technol Health Care 2007;15:433-44.

17. Joshi $A$, Weng $W$, Lichenstein $R$, et al. Prospective tracking of a pediatric emergency department e-kiosk to deliver asthma education. Health Inform J 2009:15:282-95.

18. Patton MQ. Qualitative evaluation methods. Beverly Hills, CA: Sage publications, 1980.

19. Chew LD, Bradley KA, Boyko EJ. Brief questions to identify patients with inadequate health literacy. Health 2004;11:12.

20. Bhagat M, Ghosh A. Obesity measures, metabolic profiles, blood pressure and intake of dietary fatty acids in rural women of Asian Indian origin: Santiniketan women study. J Cardiovasc Dis Res 2011:2:61.

21. Pletzke V, Henry BW, Ozier AD, et al. The effect of nutrition education on knowledge, attitude, and behavior relating to trans fatty acids in foods. Fam Consum Sci Res J 2010;39:173-83.

22. Baranowski T, Cullen KW, Nicklas T, et al. Are current health behavioral change models helpful in guiding prevention of weight gain efforts? Obes Res 2012;11(S10):23S-43S

23. Beaglehole R, Bonita R, Horton R, et al. Priority actions for the non-communicable disease crisis. The Lancet 2011;377:1438-47.

24. Esch BM, Marian F, Busato A, et al. Patient satisfaction with primary care: an observational study comparing anthroposophic and conventional care. Health Qual Life Outcomes 2008;6:74. 